\title{
DESENCADENANTES Y ESTILOS PERIODÍSTICOS DE LA INFORMACIÓN POLÍTICA EN ESPAÑA (1980-2010)
}

\section{Trigger-Factors and Journalistic Styles in the Political News in Spain (1980-2010)}

Desencadeantes e estilos jornalísticos da informação política na Espanha (1980-2010)

HUMANES, María Luisa. Universidad Rey Juan Carlos. (España)

marialuisa.humanes@urjc.es

Fecha de recibido: 29 de julio de 2015

Fecha de aceptado: 25 de septiembre de 2015

\section{RESUMEN}

El artículo analiza cómo se reflejan en el contenido de las noticias las relaciones entre actores políticos y medios de comunicación en España, tomando como referencia la teoría de la mediatización, y en concreto la mediatización de los contenidos. Se ha optado por la perspectiva de análisis longitudinal, que permite observar la evolución de estas relaciones desde la Transición hasta la actualidad. En concreto, se analizan dos indicadores de la mediatización de los contenidos: los desencadenantes de la información y los estilos periodísticos. La investigación se ha realizado a través de la técnica de análisis de contenido, aplicada a la información política en los diarios $A b c$ y El País desde 1980 a 2010. Los resultados corroboran el argumento que afirma que el modelo periodístico español se encuentra en una fase inicial del proceso de mediatización. Se concluye que la mediatización de los contenidos políticos se ha centrado en dos ejes: en el uso de estilos periodísticos alejados de la descripción y la iniciativa de publicar temas propios como desencadenantes de la información. 


\title{
ESTUDIOS
}

Palabras clave: Mediatización, prensa, España, desencadenantes informativos, estilo periodístico, información política.

\begin{abstract}
The article analyzes the relations between the political actors and the media in Spain in the news content. A longitudinal analysis is carried out to observe the evolution of these relations since the Transition to the present. Two indicators of media coverage are analyzed: the trigger-factors and the journalistic styles. The research was conducted through content analysis applied to political information in the newspapers $A b c$ and El País from 1980 to 2010. The results support the argument that the Spanish journalistic model is still in the early stage of the mediatization process. Conclusions show that the media coverage of the political system has centered on two axes: the use of journalistic styles away from the description and the initiative to publish their own news story as trigger-factors.
\end{abstract}

Keywords: Mediatization, press, Spain, trigger-factors, journalistic style, political information.

\section{RESUMO}

O artigo analisa como se refletem no conteúdo das notícias as relações entre atores políticos e meios de comunicação na Espanha, tomando como referência a teoria da mediatização, e em concreto a mediatização dos conteúdos. Se tem optado pela perspectiva de análise longitudinal, que permite observar a evolução destas relações desde a Transição até a atualidade. Em concreto, analisam-se dois indicadores da mediatização dos conteúdos: os desencadeantes da informação e os estilos jornalísticos. A pesquisa se tem realizado através da técnica de análise de conteúdo, aplicada à informação política nos jornais Abc e El País desde 1980 a 2010. Os resultados corroboram o argumento que afirma que o modelo jornalístico espanhol encontra-se em uma fase inicial do processo de mediatização. Conclui-se que a mediatização dos conteúdos políticos se tem centrado em dois eixos: no uso de estilos jornalísticos afastados da descrição e a iniciativa de publicar temas próprios como desencadeante.

Palavras-chave: Mediatização, imprensa, Espanha, desencadeantes informativos, estilo jornalístico, informação política. 


\section{La profesión periodística y el periodismo político en España en el periodo democrático}

Al finalizar la dictadura franquista en 1975 tanto el sistema político como el mediático tuvieron que poner en marcha su transición a la democracia. A partir de ese momento ambos grupos, político y periodístico, fueron de la mano a la hora de construir los cimientos de sus funciones y sus relaciones futuras como actores de una nueva esfera pública. Este artículo aborda cómo se ha reflejado en los contenidos de la información política las relaciones entre actores políticos y medios de comunicación en España. La hipótesis de partida se centra en el argumento de que el periodismo español, desde el final de la dictadura, ha ido adquiriendo las rutinas de producción de la información política propias del proceso de la mediatización, es decir, ha incrementado su independencia respecto a los actores políticos y como consecuencia influye en las instituciones políticas (Strömbäck, 2008). Como afirman Mazzolleni y Schutz (1999):

Mediatization is, in fact, a phenomenon that is common to the political systems of almost all democratic countries, where it has taken different shapes and developed at different speeds. However, it has in all cases proved impossible to contain because the media have assumed the character of "necessity" in the political domain (p. 249).

El estudio de la mediatización en el caso español es especialmente interesante puesto que la pertenencia del sistema de medios en España al modelo pluralista-polarizado implica a nivel teórico, al menos, que el proceso de mediatización -y los términos asociados de intervencionismo e independencia mediática- estaría frenado por el paralelismo político como característica propia de las relaciones entre medios y políticos. Como han señalado Strömbäck y Dimitrova (2006) las culturas periodísticas nacionales son un elemento moderador del proceso de mediatización del contenido noticioso. Algunos estudios comparados recientes (Strömbäck \& Luengo, 2008; Strömbäck et al., 2011; van Dalen, de Vreese \& Albaeck, 2012) muestran efectivamente que el caso español está aún lejos de los niveles de mediatización de los contenidos encontrados en los medios de los países del modelo liberal y corporativo.

Al inicio de los años 70, aun cuando la dictadura estaba ya muy debilitada, la profesión periodística se encontraba en una situación de inferioridad frente al poder político por las enormes restricciones que imponía el marco legal al ejercicio del periodismo, que se concretaron en el cierre de publicaciones y las sanciones contra los periodistas adversos al régimen. El periodismo español vivió desde finales de la década de los años 70 una serie de cambios transcendentales después de décadas de control férreo del régimen franquista. Se derogó la Ley de Prensa de 1966 dando paso a la plena libertad de expresión reconocida constitucionalmente; se consolidaron nuevos grupos empresariales y desapareció la cadena de prensa del Movimiento; se liberalizó la profesión y se redefinieron las asociaciones de la Prensa y otras organizaciones profesionales. Las redacciones de los medios de comunicación vieron llegar a una nueva generación de jóvenes periodistas, que sustituirán a los redactores que se vincularon a la profesión en la postguerra. Este relevo generacional estuvo motivado por razones de tipo político, que impedían en muchos casos a los antiguos periodistas adaptarse a las nuevas condiciones de la prensa.

Después de años de subordinación, los periodistas españoles tuvieron la oportunidad de iniciar un proceso de profesionalización que les condujera hacia mayores niveles de autonomía frente al poder político. En frente tenían 
a una clase política que también necesitaba legitimarse en el nuevo sistema democrático, y para ello requerían la colaboración de los medios de comunicación. Durante la Transición los medios y la nueva clase política entraron en una fase de cooperación, consecuencia de la evolución paralela que siguieron ambos grupos después de la Dictadura para mejorar su prestigio social (Casero, 2012, p. 32). La prensa debía hacer desaparecer la imagen de mediocridad en la que estuvo sumida durante el Franquismo y los políticos debían legitimarse en el sistema democrático.

Como han señalado Gunther, Montero y Wert (1999, pp. 14-17) la mayoría de los medios sirvieron como correa de transmisión de las decisiones políticas del gobierno presidido por Adolfo Suárez y como altavoz del discurso de los partidos políticos y los sindicatos frente a la opinión pública. Pero quizás la función más importante fue la de agentes de socialización política y promotores de los valores democráticos. La debilidad de las élites políticas convirtió a los periodistas en una pieza clave en la recién nacida democracia y comenzaron a adquirir un gran prestigio. La percepción del importante papel de los medios en el proceso de democratización se confirma si se tienen en cuenta los datos del estudio del Centro de Investigaciones Sociológicas, titulado Transición y democracia (1996). En este informe los españoles encuestados sitúan a la prensa en el quinto lugar entre los grupos que intervinieron de manera significativa en la transición a la democracia. Sin duda, en la memoria colectiva ha quedado fijado el importante papel que cumplieron los medios durante el intento de golpe de Estado el 23 de febrero de 1981. De hecho se conoce lo sucedido como "noche de los transistores" (Humanes, 1998). En resumen,

los medios se apropiaron en esta época de funciones propias de otras instituciones del sistema democrático, sustitución que tiene su origen en la propia debilidad de estas instituciones y que determinan la naturaleza de las relaciones de los periodistas y los políticos en la actualidad. Se asistió a la consolidación de los periodistas como actores políticos, ejerciendo este papel a través de varios mecanismos: la representación directa de la opinión pública, la capacidad de legitimar determinados aspectos de la vida social, la fijación de normas de conducta y el "otorgamiento de notoriedad" (Ortega, 1994, pp. 43-44).

Después de 1982, los periodistas mantuvieron y aumentaron su prestigio, esta vez, distanciándose del poder político. Gracias a este rol activo y crítico, la profesión periodística llegó a ocupar el primer lugar en prestigio en comparación con otros grupos e instituciones; justo en el momento en el que precisamente se publican los escándalos político-económicos que más interés han despertado, y que salieron a la luz a través de los medios de comunicación. Ortega $(2003,2011)$ propone el concepto de "antagonistas complementarios" como modo de relación básica entre políticos y periodistas en estos años.

Las nuevas relaciones entre políticos y periodistas al final del período de los gobiernos socialistas, presididos por Felipe González, tienen como consecuencia importante el surgimiento de la polarización política y mediática (González, 2008; González \& Bouza, 2009) y el reforzamiento del paralelismo político (Castells 2009), propio del modelo pluralista polarizado (Hallin y Mancini, 2004). Desde la década de los años 90 "la cercanía con el poder - la oposición ha marcado el desarrollo de los grandes grupos de comunicación" (Díaz Nosty, Roto, \& Urbaneja, 2011, p. 20). Diversos estudios confirman que el periodismo político español está altamente politizado y que responde a alineamientos ideológicos con una creciente polarización (Casero, 2012; González \& Novo, 2012; Martínez \& Humanes, 2012; van Dalen, de Vreese \& Albaeck, 2012). Las tensiones entre medios y partidos políticos se han concretado en los conflictos motivados por las sucesivas concesiones de licencias en el sector audiovisual a partir de los años 90 y las ayudas a la prensa en sus distintas variantes (publicidad, subvenciones). En segundo término, la consolidación de un periodismo orientado al comentario, y vehiculado a través de las tertulias radiofónicas y 
televisivas, en el que los periodistas actúan como representantes de posiciones ideológicas coincidentes con las de los partidos políticos (Ortega, 2006).

El caso español se ajustaría a lo señalado por Mazzoleni (2010) cuando afirma que en algunos contextos el papel de adversario de los medios no se corresponde con la defensa de los intereses generales, sino que es una estrategia para actuar políticamente (p. 99). Van Dalen, Albæk y de Vreese (2011) afirman a partir de los datos de una encuesta realizada a periodistas que cubren la información política en Alemania, Reino Unido, Dinamarca y España, que los periodistas españoles muestran una aptitud más cínica hacia los políticos que sus otros colegas como forma de eludir las presiones por parte del poder político. En un estudio reciente, las entrevistas en profundidad a jefes de la sección de información política de 14 medios españoles han desvelado que las actitudes más presentes al definir las relaciones actuales con los políticos son la desconfianza y el distanciamiento (Humanes, Martínez \& Saperas, 2013).

En este trabajo se analizará cómo se reflejan, en el contenido de las noticias, las relaciones entre actores políticos y los medios de comunicación, tomando como referencia la teoría de la mediatización, en concreto la mediatización de los contenidos. Hemos optado por la perspectiva de análisis longitudinal, que nos permita observar la evolución de estas relaciones desde la Transición hasta la actualidad. Puesto que el proceso de mediatización es lento, su análisis ha de realizarse a lo largo de décadas (Kepplinger, 2002). En concreto, abordaremos el estudio de dos indicadores de la mediatización de los contenidos: los desencadenantes de la información y los estilos periodísticos.

\section{La mediatización de los contenidos políticos}

El concepto de mediatización implica la idea de un proceso de cambio que tiene como consecuencia el incremento de la independencia de los medios y de su influencia en otras instituciones, especialmente en las políticas. Strömbäck (2008) establece cuatro fases o dimensiones en el proceso de mediatización: en la primera los medios constituyen la fuente más importante de información; en la segunda los medios son más independientes respecto a otras instituciones; en la tercera el contenido de los medios se rige por la lógica mediática más que por la lógica política, y finalmente, los actores políticos se rigen por la lógica mediática.

En el proceso de mediatización, el sistema político pierde autonomía en la medida en que el sistema periodístico tiene más capacidad de influir sobre la política (Mazzoleni y Schutz, 1999, p. 250). Esta capacidad de intervenir de los medios sobre la política se lleva a cabo a través de ciertas rutinas y prácticas periodísticas (Strömbäck \& Esser, 2009). En primer lugar, aplicando determinados criterios de selección de las noticias (news-value). En segundo lugar, construyendo una esfera pública mediática. En tercer lugar, imponiendo la lógica mediática sobre cualquier otra forma de discurso político. Al final del proceso de mediatización el periodista aumenta su nivel de profesionalidad, adopta una actitud más pragmática y asume más control sobre la producción de los contenidos noticiosos (Strömbäck, 2008).

Este artículo se centrará en analizar, en primer lugar, si a lo largo del periodo de tiempo considerado los medios de comunicación han modificado los criterios de selección de los acontecimientos noticiosos. En este sentido partimos de la distinción realizada por Strömbäck y Nord (2006) entre 1) los procesos de news making y construcción de la agenda y 2) los procesos de determinación del contenido y del tratamiento de las noticias (framing), para abordar 
el primero de ellos: cuál de los dos grupos - políticos o periodistas - tiene mayor capacidad para desencadenar (o promover) los ítems informativos en el proceso de negociación de la noticiabilidad, señalado por Cook (1998).

El desencadenante y el contenido de la información no siempre son lo mismo, sino que como señala Kepplinger (2002) los trigger-factors son los estímulos que dan lugar a una noticia, mientras que el contenido es el tópico sobre el que se informa (p. 975). Este autor divide los factores desencadenantes en tres tipos: events (acontecimientos concretos como la aprobación de una ley, por ejemplo), statements (declaraciones de los actores sobre acontecimientos, pero con la intención de interpretarlos desde su punto de vista como fuente) y los issues (temas a largo plazo). En su estudio sobre la mediatización de las actividades del parlamento alemán, Kepplinger (2002) concluyó que aunque la mayoría de las noticias estaban originadas por events, las declaraciones habían tenido un gran incremento a lo largo del periodo considerado (1951-1995), lo que se interpreta como una consecuencia del proceso de mediatización. Otros autores corroboran el argumento de que las noticias políticas originadas por acontecimientos relacionados con las políticas públicas se han visto relegadas por aquellos eventos diseñados y programados por los políticos (Livingstone \& Bennett, 2003; Walgrave \& van Aelst, 2006). Sin embargo, en un contexto periodístico similar al español, Mellado y Rafter (2014) encontraron en su análisis de la información política en Chile que los events (eventos) originaban la mayor parte de las noticias frente a los statements (declaraciones) o issues (temas), y además su presencia se incrementó en la prensa chilena entre 2006 y 2011.

A partir de estas evidencias se han planteado dos hipótesis referentes a los factores desencadenantes como indicador del carácter de las relaciones entre actores políticos y medios en el caso español:

H1: A lo largo del tiempo disminuirán los desencadenantes basados en acontecimientos y se incrementarán las noticias desencadenadas por declaraciones y eventos programados por los actores políticos como reflejo de un mayor ajuste de la acción política a la lógica mediática.

H2: A lo largo del tiempo se incrementarán las informaciones basadas en temas propios en los diarios El País y $A b c$ como consecuencia de haber conseguido mayor independencia respecto a los actores políticos.

El otro indicador del grado de mediatización de los contenidos de los medios es el estilo periodístico utilizado por el periodista en la cobertura de la realidad política (Strömbäck \& Dimitrova, 2011, p. 36). En este sentido se distingue entre dos estilos, el descriptivo y el interpretativo. Cuando predomina el estilo descriptivo, el periodista toma una actitud pasiva, de mero transmisor de la información generada por los actores políticos. El estilo interpretativo, por su parte, da más protagonismo al periodista, que no sólo relata los hechos, sino que además los explica y los contextualiza. Este estilo periodístico más interpretativo y crítico es un indicador de mediatización de los contenidos (Strömback \& Dimitrova, 2011).

La literatura muestra consenso en afirmar que desde la década de los años 60 el estilo periodístico interpretativo ha incrementado su presencia frente al periodismo descriptivo (Blumler \& Gurevitch, 1995; Patterson, 1996; Benson \& Hallin, 2007; Salgado \& Strömbäck, 2013; Strömbäck, 2008; Strömbäck \& Aalberg, 2008; Strömbäck \& Dimitrova, 2006; Strömbäck \& Shehata, 2007). Encontramos dos interpretaciones para explicar por qué se ha generalizado la tendencia a recurrir al estilo interpretativo en todos los modelos periodísticos. Por una parte, para algunos autores el estilo interpretativo es un indicador de mayor independencia del periodista respecto al sistema político (Patterson, 2000; Strömbäck \& Dimitrova, 2006). Para Patterson (2000): 
The interpretive style empowers journalists by giving them more control over the news message. Whereas descriptive reporting is driven by the facts, the interpretive form is driven by the theme around which the story is built. Facts become the materials with which the chosen theme is illustrated (...) The descriptive style places the journalist in the role of an observer. The interpretive style requires the journalist to act also as an analyst. The journalist is thus positioned to give shape to the news in a way the descriptive style does not allow (p. 250).

Por otra parte, McNair (2000) añade que el estilo interpretativo es también consecuencia de la creciente comercialización y competencia entre medios, de modo que se convierte en una estrategia comercial más.

La tercera hipótesis plantea que el periodista español ha adoptado desde el final de la dictadura este estilo más activo en la cobertura noticiosa de la política, incrementando el uso de la interpretación y la crítica:

H3: Los periódicos españoles han incrementado el uso de un estilo periodístico orientado a la interpretación y a la crítica.

Finalmente se ha formulado una última hipótesis en la que se plantea la asociación entre los distintos desencadenantes y el estilo periodístico:

H4: El desencadenante de la información estará relacionado con el estilo periodístico, de tal forma que cuando el desencadenante sea el propio diario, el estilo periodístico será menos descriptivo y más orientado a la interpretación y la crítica.

\section{Metodología}

La investigación se ha realizado a través de la técnica de análisis de contenido, aplicada a la información política en los diarios Abc y El País desde 1980 a 2010. El diseño muestral se ha desarrollado en tres fases. Primero se seleccionaron los diarios de los cuales se extrajo posteriormente la muestra, se eligieron Abc y El País porque son los dos únicos periódicos que se han editado durante los 30 años analizados; además representan dos líneas editoriales claramente diferencias. $A b c$, que comienza a editarse en 1905, es un diario conservador y monárquico; $E l$ País nace en 1976 con una tendencia ideológica que se sitúa en la centro-izquierda, progresista y socialdemócrata.

En la segunda fase se seleccionaron dos años, en periodos de cinco años, por cada década (1980-1985; 1990-1995; 2000-2005-2010). En la tercera y última fase se han extraído aleatoriamente dos semanas naturales de cada año, cada una correspondiente a un semestre. Se ha analizado, por tanto, la información política publicada por estos periódicos en 14 semanas completas.

Como unidad de análisis se consideró cada texto (noticia, reportaje, breve, entrevista, artículo de opinión y fotografía informativa) publicado en la sección: Nacional/España/Política de cada diario, para un total de 3802 unidades (incluidos los textos de opinión, que fueron descartados del análisis de contenido). De acuerdo a los objetivos del trabajo quedaron excluidos de la codificación tanto los textos de opinión (incluidas las viñetas) como las fotografías informativas. Se excluyeron también las informaciones referentes a procesos electorales, puesto que los objetivos del trabajo se centran en la información política en periodos de actividad política rutinaria. Para 


\section{ESTUDIOS}

Walgrave y van Aelst (2006) los actores políticos y los periodistas siguen una lógica distinta en periodo electoral a la que siguen en periodo de política ordinaria: a) cambia el comportamiento de los actores políticos, que intentan llamar más la atención de los medios; b) los medios tienen más problemas para establecer su propia agenda de temas, y c) los medios en periodo electoral se rigen por unas reglas que promueven la equidad y el juego limpio. En total se analizaron 3174 textos de los dos diarios. La obtención de los datos fue realizada por dos codificadores, que fueron previamente entrenados en los criterios de codificación. Para determinar la fiabilidad del proceso, de manera aleatoria, se codificó doblemente el $10 \%$ de las unidades de análisis. El cálculo de la pi de Scott fijó un valor medio de acuerdo entre codificadores del 0,8. En la tabla 1 se encuentran los datos de identificación básicos de la muestra.

Tabla 1. Datos de identificación de la muestra (\%)

\begin{tabular}{|c|c|}
\hline \multicolumn{2}{|l|}{ Diario } \\
\hline$A b c$ & 46 \\
\hline El País & 54 \\
\hline Aparición en la portada & 31,3 \\
\hline \multicolumn{2}{|c|}{ Género } \\
\hline Noticia & 53,3 \\
\hline Reportaje & 22,7 \\
\hline Breve & 6,4 \\
\hline Entrevista & 1,4 \\
\hline Análisis-artículo de opinión & 10,1 \\
\hline Fotografía informativa & 6,3 \\
\hline \multicolumn{2}{|c|}{ Autoría } \\
\hline Redactor del medio & 61,1 \\
\hline Agencia & 6,9 \\
\hline Redacción & 17 \\
\hline Otros medios & 0,1 \\
\hline Colaborador externo al medio & 2,9 \\
\hline No identificable & 12.2 \\
\hline Total & 3802 \\
\hline
\end{tabular}

Fuente: elaboración propia. 
La ficha de codificación general contiene 37 variables, clasificadas en seis apartados: datos de identificación del texto e indicadores relacionados con el tema de la información, los actores del relato, los escenarios de la acción política, los desencadenantes y el estilo periodístico (de los que solo se utilizaron para el análisis los datos relativos a los dos últimos).

Las variables utilizadas para el desarrollo de este artículo se han operacionalizado como sigue. Los desencadenantes de la información se han codificado a través de estas categorías:

$1=$ Acontecimiento (discusiones en el pleno del congreso, discusiones sobre el presupuesto, tratados internacionales, presentación de un decreto). Se corresponde con los event-driven news (Livingston \& Bennet, 2003) o los events (Kepplinger, 2002).

$2=$ Noticia desencadenada por un actor político (declaración, rueda de prensa). Diseñadas y programadas por los políticos en un escenario institucional, en el sentido de Boorstin (1977).

$3=$ Decisión interna al medio.

$4=$ Reacción del actor político a lo publicado por un medio.

$5=$ Reacción del actor político a lo publicado/dicho por otro actor político.

$6=$ No identificable.

El posicionamiento del periodista respecto al actor/acción política se ha codificado a través de cuatro categorías:

$1=$ Descriptivo (se cuenta lo que sucede).

$2=$ Interpretativo (se analiza y evalúa la situación mientras se describe).

$3=$ El periodista destapa las intenciones del político cuando informa sobre su actividad política (Zaller, 1999).

4= El periodista ejerce la crítica sobre la acción política. Aparece la opinión del periodista.

\section{Resultados}

La primera hipótesis prevé que con el transcurso del tiempo los desencadenantes basados en acontecimientos (events) disminuyen, mientras se produce la tendencia contraria con las noticias desencadenadas por declaraciones y eventos programados por los actores políticos. Los datos (tabla 2) han corroborado solo parcialmente la hipótesis planteada, puesto que si bien es cierto que a partir de 1995 hay un descenso de las noticias basadas en acontecimientos, también lo ha habido en las informaciones que tienen como desencadenante las declaraciones de los actores políticos. Hay que destacar, no obstante, que se han producido incrementos estadísticamente significativos en las noticias cuyos desencadenantes son reacciones de los actores políticos ante declaraciones de otros políticos o de los medios de comunicación.

Cuando se ha considerado si estas tendencias son similares en los dos diarios, los resultados han revelado que en el primer año del análisis (1980) no existían diferencias estadísticamente significativas entre $A b c$ y El País, pero a partir de 1985 sí se dan. Más exactamente, el análisis de los residuos tipificados corregidos indica que las diferencias se concentran en las categorías: noticias desencadenadas por acontecimientos y declaraciones de los actores políticos. Así en el diario El País hay una presencia mayor de las noticias desencadenadas por acontecimientos (55,7\% en $1990,50,4 \%$ en $1995,52 \%$ en $2000,66,4 \%$ en 2005 y $42,1 \%$ en 2010$)$, mientras que en $A b c$ son mayores 
los porcentajes de las declaraciones de los actores políticos en los años analizados (48,5\% en 1990, 41,4\% en 1995, $55,7 \%$ en $2000,45,4 \%$ en 2005 y $43,1 \%$ en 2010 ).

Tabla 2. Evolución de los tipos de desencadenantes en los dos diarios (\% y residuos tipificados corregidos ${ }^{1}$ )

\begin{tabular}{|c|c|c|c|c|c|c|c|}
\hline & 1980 & 1985 & 1990 & 1995 & 2000 & 2005 & 2010 \\
\hline \multirow{2}{*}{ Acontecimientos } & 41,7 & 37,0 & 40,0 & 31,8 & 37,2 & 38,1 & 34,6 \\
\hline & 1,9 &,- 2 & 1,3 & $-2,7$ &,- 1 & ,4 &,- 7 \\
\hline \multirow{2}{*}{$\begin{array}{l}\text { Desencadenado por la declaración de } \\
\text { un actor político }\end{array}$} & 42,7 & 37,5 & 37,5 & 36,8 & 44,2 & 36,8 & 25,5 \\
\hline & 1,8 &,- 5 &,- 5 &,- 8 & 3,0 &,- 9 & $-3,4$ \\
\hline \multirow{2}{*}{ Tema propio del medio } & 6,3 & 13,4 & 13,3 & 15,9 & 11,1 & 13,1 & 24,8 \\
\hline & $-4,2$ & ,3 & ,3 & 2,0 & $-1,4$ & ,1 & 4,5 \\
\hline \multirow{2}{*}{$\begin{array}{l}\text { Reacción del actor político a lo } \\
\text { publicado por un medio }\end{array}$} & ,5 & 9 & 1,5 &, 7 & ,4 & ,2 & 7,8 \\
\hline & $-1,1$ &,- 3 & 1,0 &,- 9 & $-1,7$ & $-2,1$ & 8,5 \\
\hline \multirow{2}{*}{$\begin{array}{l}\text { Reacción del actor político a lo } \\
\text { publicado/dicho por otro actor político }\end{array}$} & 2,5 & 5,2 & 1,5 & 2,4 & 4,3 & 6,2 & 3,9 \\
\hline & $-1,4$ & 1,7 & $-2,8$ & $-1,7$ & ,6 & 3,2 & ,1 \\
\hline \multirow{2}{*}{ No identificables } & 6,3 & 5,9 & 6,1 & 12,4 & 3,0 & 5,6 & 3,3 \\
\hline & , 0 &,- 3 &,- 1 & 5,9 & $-3,5$ &,- 7 & $-1,6$ \\
\hline
\end{tabular}

Fuente: elaboración propia.

$X^{2}=194,379 \mathrm{gl} 30 \mathrm{p} \leq, 000$

La segunda hipótesis aventuraba el incremento de las informaciones basadas en temas propios de los dos diarios como consecuencia de la mayor independencia conseguida por los periodistas respecto a los actores políticos desde el inicio de la Transición hasta la actualidad. Efectivamente, en la tabla 2 se puede observar que de 1980 a 2010 se ha multiplicado por cuatro el porcentaje de informaciones en las que los dos diarios han tomado la iniciativa. Esta tendencia es, además, similar en los dos diarios.

En consonancia con el argumento de la mediatización creciente de los contenidos noticiosos, la tercera hipótesis plantea que en el periodo de tiempo considerado habrá un incremento del estilo periodísticos más interpretativo y crítico. Como reflejan los resultados de la tabla 3, aunque sigue predominando claramente el estilo descriptivo en el conjunto de la muestra, existe una clara tendencia hacia el menor uso de la mera descripción en la cobertura de la política a favor de los otros tres estilos periodísticos. La perspectiva longitudinal de este estudio nos permite explicar mejor los resultados de investigaciones centradas en un punto concreto del tiempo

1 Los valores z (residuos tipificados corregidos) muestran qué casillas de la tabla de contingencia son las responsables de la asociación. En este caso se consideraron estadísticamente significativos los residuos con valores $\geq 1,96$. 
-especialmente la información durante las campañas electorales-, que afirman la escasa presencia del estilo interpretativo en el periodismo español. Por ejemplo Strömbäck y Luengo (2008) encontraron que solo el 38,7\% de las noticias en los diarios españoles durante la campaña electoral de 2008 seguían el estilo interpretativo. También Strömbäck et al. (2011) afirman que en España solo un 9,5\% de las noticias de televisión sobre la campaña electoral al parlamento europeo se ajustaban al estilo interpretativo.

Tabla 3. Evolución temporal de los estilos periodísticos de los dos diarios (\% y residuos tipificados corregidos)

\begin{tabular}{|l|c|c|c|c|c|c|c|}
\hline \multirow{3}{*}{ Descriptivo } & \multicolumn{1}{|c|}{1980} & 1985 & 1990 & 1995 & 2000 & 2005 \\
& 76 & 75,6 & 68,2 & 62,5 & 57,3 & 55,7 & 36,0 \\
\cline { 2 - 8 } & 6,2 & 6,3 & 3,0 &, 2 & $-2,6$ & $-3,3$ & $-10,3$ \\
\hline \multirow{2}{*}{ Interpretativo } & 13,2 & 15,0 & 18,7 & 29,0 & 29,8 & 28,1 & 34,4 \\
\cline { 2 - 9 } & $-5,4$ & $-4,8$ & $-3,0$ & 2,6 & 3,4 & 2,3 & 4,6 \\
\hline \multirow{2}{*}{ Crítico } & 1,0 & 2,2 & 6,1 & 4,6 & 5,9 & 9,4 & 13,9 \\
\hline \multirow{2}{*}{ Desvela intenciones del político } & $-4,5$ & $-3,6$ & -1 & $-1,4$ &,- 1 & 3,6 & 6,3 \\
\cline { 2 - 9 } & 9,4 & 7,2 & 6,9 & 3,9 & 7,0 & 6,8 & 15,7 \\
\cline { 2 - 9 } & 1,3 &,- 5 &,- 7 & $-3,3$ &,- 7 &,- 9 & 5,7 \\
\hline
\end{tabular}

Fuente: elaboración propia.

$X^{2}=252,526 \cdot g l 18 p \leq, 000$

Los residuos tipificados corregidos de la tabla 3 nos muestran que la tendencia hacia un periodismo más interpretativo comienza a cambiar a partir de la segunda mitad de los años 90 . Si comparamos el estilo descriptivo con el interpretativo, el cambio se manifiesta en los datos para la información analizada en los años 1995 y 2000, coincidiendo con el momento señalado por algunos autores como la culminación de las relaciones de cooperación entre medios y políticos que había caracterizado al periodo de la Transición. Se corrobora también el argumento de la comercialización expuesto por McNair (2000) que afirmaba que la competencia entre medios y la comercialización tenía como consecuencia recurrir al estilo interpretativo como estrategia comercial. En este sentido, hay que señalar que la televisión comercial privada en España comienza sus emisiones en 1990, y que por lo tanto, es lógico que sus efectos sobre la prensa escrita se hagan notar precisamente en los años señalados. De hecho, los datos que poseemos sobre el uso de los estilos descriptivo e interpretativo en la información televisiva en España nos indican que el $36,9 \%$ de las noticias recurren a la interpretación, subiendo el porcentaje hasta más del $40 \%$ en las cadenas de televisión privadas (Humanes et al., 2013).

La tabla 3 muestra también lo que podríamos considerar como una tercera etapa en las relaciones entre medios y políticos a partir de 2005, donde se destaca el incremento de la crítica y la actitud por desenmascarar las intenciones del político y ponerlas de manifiesto frente a la ciudadanía. 
Cuando se considera la evolución de los estilos periodísticos por separado en los dos diarios, observamos que la tendencia general se cumple para los dos, es decir, tanto el diario Abc como El País han recurrido en las últimas décadas a un estilo más interpretativo y menos descriptivo. No obstante, existen diferencias en las dos publicaciones. En primer lugar, El País partía en 1980 con un mayor porcentaje de informaciones con estilo interpretativo que $A b c$, sin duda reflejo del deseo de sus promotores de instaurar un modelo periodístico distanciado de las formas de hacer periodismo de los diarios que habían estado bajo el rígido control de las leyes de prensa franquistas - más apegados al periodismo descriptivo cuya función principal es la de vehicular mensajes desde el sistema político a la ciudadanía- (Mazzoleni \& Schutz, 1999).

En la comparación de los estilos descriptivo e interpretativo en los dos diarios para el año 2010, se encuentra que no existen diferencias estadísticamente significativas $\left(X^{2}=5,399 p \leq, 145\right)$. Para los otros dos estilos (crítico y desenmascarador) se evidencia en 1980 una situación similar a la ya descrita. Mientras que en Abc en 1980 prácticamente no hay presencia de los estilos crítico y desenmascarador, El País muestra un porcentaje mayor de informaciones siguiendo estos dos estilos. A pesar de estas diferencias en los primeros momentos de la Transición, en 2010 tampoco se encuentran diferencias estadísticamente significativas $(X 2=5,399 p \leq, 145)$.

Por último, en la cuarta hipótesis se planteó que el desencadenante de la información está relacionado con el estilo periodístico, de tal forma que cuando el desencadenante es el propio diario el estilo periodístico será menos descriptivo y más orientado a los otros tres estilos. Los resultados indican que no se han producido cambios durante el periodo de tiempo considerado en la relación entre tipos de desencadenantes y estilos periodísticos, por lo que se trabajará con los datos globales de la muestra. Como se observa en la tabla 4 (con los datos de toda la muestra), el análisis de residuos tipificados corregidos corrobora la hipótesis e indica dos tendencias importantes. Primero, una sobrerrepresentación del estilo descriptivo cuando los desencadenantes son acontecimientos o declaraciones de un actor político. Segundo, la fuerte presencia de los estilos más alejados de la descripción cuando es el medio de comunicación el que toma la iniciativa en la publicación. Los dos diarios muestran la misma tendencia. 


\section{ESTUDIOS}

Tabla 4. Relación entre tipos de desencadenantes y el estilo periodístico en los dos diarios (\% y residuos tipificados corregidos)

\begin{tabular}{|c|c|c|c|c|}
\hline \multirow[t]{2}{*}{ Desencadenante } & \multicolumn{4}{|c|}{ Estilo periodístico } \\
\hline & Descriptivo & Interpretativo & $\begin{array}{l}\text { Desvela } \\
\text { intenciones del } \\
\text { actor político }\end{array}$ & Crítico \\
\hline \multirow{2}{*}{ Acontecimientos } & 39,7 & 37,0 & 28,0 & 7,9 \\
\hline & 3,2 &,- 4 & $-3,0$ & $-2,6$ \\
\hline \multirow{2}{*}{ Desencadenado por un actor político } & 41,6 & 36,5 & 29,0 & 29,1 \\
\hline & 4,1 & $-1,4$ & $-3,1$ & $-2,6$ \\
\hline \multirow{2}{*}{ Decisión interna del medio } & 8,0 & 15,8 & 27,1 & 35,2 \\
\hline & $-10,2$ & 2,8 & 6,6 & 8,9 \\
\hline \multirow{2}{*}{$\begin{array}{l}\text { Reacción del actor político a lo publicado } \\
\text { por un medio }\end{array}$} & 1,0 & 1,4 & ,9 & 0 \\
\hline &,- 4 & 1,3 &,- 1 & $-1,3$ \\
\hline \multirow{2}{*}{$\begin{array}{l}\text { Reacción del actor político a lo publicado/ } \\
\text { dicho por otro actor político }\end{array}$} & 4,2 & 4,0 & ,9 & 1,2 \\
\hline & 1,7 & ,4 & $-2,2$ & $-1,8$ \\
\hline \multirow{2}{*}{ No identificables } & 5,6 & 5,2 & 14,0 & 6,7 \\
\hline & $-1,7$ & $-1,2$ & 4,9 & ,3 \\
\hline
\end{tabular}

Fuente: elaboración propia.

$X^{2}=198,859 \mathrm{gl} 15 \mathrm{p} \leq, 000$

\section{Conclusiones}

El objetivo principal de este artículo era estudiar cómo se ha desarrollado el proceso de mediatización en el periodismo en España a través del análisis de la información política publicada en los diarios El País y Abc entre 1980 y 2010. Para ello se han tomado dos indicadores de mediatización: los desencadenantes de la información y el estilo periodístico. La literatura señala que uno de los resultados del proceso de mediatización es una mayor independencia de los medios y de los periodistas respecto a los actores políticos. En concreto, en el caso de las dos variables consideradas, si teóricamente se cumple esta hipótesis se debería producir un descenso de los desencadenantes basados en declaraciones de los políticos (Kepplinger, 2002) y en actividades programadas para la cobertura mediática (Livingstone \& Bennett, 2003; Walgrave \& van Alelst, 2006). Los actores políticos harían un esfuerzo para adaptarse a la lógica de los medios, propia de la última fase del proceso de mediatización (Strömbäck, 2008). También se afirma que el estilo periodístico interpretativo está relacionado con mayores niveles de mediatización de los contenidos (Strömbäck \& Dimitrova, 2011). 
En el caso español, la tradición de un periodismo fuertemente caracterizado por el paralelismo político propio del modelo pluralista polarizado apuntaría a que el nivel de mediatización será aún bajo. No obstante, en trabajos anteriores se ha encontrado que a partir de la década del 2000 otros indicadores de mediatización se han incrementado significativamente (Martínez, Humanes \& Saperas, 2014). Los resultados del presente trabajo corroboran el argumento que afirma que el modelo periodístico español se encuentra en una fase inicial del proceso de mediatización.

En cuanto a los desencadenantes, de manera general siguen predominando los acontecimientos. Las declaraciones de los políticos en las tres categorías en las que se han operacionalizado -la noticia desencadenada por un actor político mediante una declaración, una rueda de prensa, etc.; la reacción del actor político a lo publicado por un medio, y la reacción del actor político a lo publicado/dicho por otro actor político- no muestran una tendencia clara hacía una mayor presencia, salvo en las declaraciones motivadas por la reacción a lo publicado por otro actor político, lo cual encaja con el incremento del conflicto como encuadre informativo (Martínez, Humanes \& Saperas, 2014). El patrón más claro es el incremento continuado en el tiempo de los temas propios del medio (hipótesis 2), es decir, de aquellas noticias que no tienen origen en instancias externas al medio. Este es el indicador más preciso de independencia respecto al sistema político. Por lo tanto, la primera hipótesis se ha corroborado parcialmente.

Cabe destacar las diferencias entre El País y Abc respecto a la importancia dada a los desencadenantes. El País durante todo el periodo mantiene los acontecimientos como desencadenante fundamental de la información política, mientras que $A b c$ prioriza las declaraciones de los políticos. En el resto de categorías las diferencias entre los dos periódicos no existen.

La tercera hipótesis se ha corroborado totalmente: el incremento de los estilos periodísticos alejados de la mera descripción es constante, principalmente a partir del año 2000. Los dos diarios, además, tienden a recurrir a estos estilos de manera semejante en la última década.

Por último, se pretendía analizar la relación entre los estilos periodísticos y los desencadenantes. Los datos han indicado que existe una asociación estadísticamente significativa entre los indicadores, pero esta relación es más fuerte entre el estilo descriptivo y los acontecimientos como desencadenantes, por un lado, y las noticias promovidas por el medio con las tres categorías que reflejan un papel activo del periodista (estilo interpretativo, intención de desvelar las intenciones del actor político y actitud crítica), por el otro. No se han hallado diferencias ni cuando se ha considerado la variable temporal ni cuando se han comparado los dos diarios.

Los resultados, gracias al análisis longitudinal, permiten conocer cómo el modelo de periodismo político en España se ha transformado desde los años 80, especialmente a partir del año 2000 se va ajustando de manera progresiva a las características de otros modelos periodísticos que se encuentran en fases de mediatización más avanzadas. Se puede concluir que la mediatización de los contenidos políticos en el caso de la prensa española se ha centrado en dos ejes: el uso de estilos periodísticos alejados de la descripción y la iniciativa de publicar temas propios. Además, estas dos características son las que presentan una relación más clara entre sí, como indican los residuos tipificados corregidos (tabla 4).

No obstante, dado que el inicio del siglo xxı marca un cambio, sería conveniente replicar el análisis incluyendo diarios como El Mundo y La Razón, que comenzaron a publicarse con posterioridad, así como los nuevos diarios nativo digitales, para profundizar más en el conocimiento del proceso de mediatización de la prensa española. También se requeriría ampliar la muestra incluyendo los medios audiovisuales. 


\section{Referencias}

1. Benson, R., \& Hallin, D. C. (2007). How states, markets and globalization shape the news: The French and US National Press, 1965-97. European Journal of Communication, 22(1), 27-48.

2. Blumler, J. G., \& Gurevitch, M. (1995). The crisis of public communication. London: Routlegde.

3. Boorstin, D. (1977). The Image. Nueva York: Atheneum.

4. Casero, A. (2012). El periodismo político en España: algunas características definitorias. Periodismo político en España: concepciones, tensiones y elecciones. La Laguna: Sociedad Latina de Comunicación Social, (pp. 19-46).

5. Castells, M. (2009). Comunicación y poder. Madrid: Alianza editorial.

6. Cook, T. (1998). Governing with the news: the news media as a political institution. Chicago University Press.

7. Díaz Nosty, B., Roto, E., \& Urbaneja, F. G. (2011). Libro negro del periodismo en España. Madrid: Asociación de la Prensa de Madrid.

8. González, J. J. (2008). Los medios en la democracia avanzada. Hacia una democracia de audiencia. Tres décadas de cambio social en España. Madrid: Alianza.

9. González, J. J., \& Bouza. F. (2009). Las razones del voto en la España democrática 1977-2008. Madrid: Catarata.

10. González, J. J., \& Novo, A. (2012). The role of the media agenda in a context of political polarization. Comunicación y Sociedad, 24(2), 131-147.

11. Gunther, R., Montero, J. R., \& Wert, J. I. (1999). The media and politics in Spain: from dictatorship to democracy. WP n 176, Barcelona: Institut de Ciencies Polítiques i Socials.

12. Hallin, D., \& Mancini, P. (2004). Comparing media systems. Three models of media and politics. Cambridge University Press.

13. Humanes, M. L. (1998). La profesión periodística en España. Zer: Revista de estudios de comunicación 3(4), 265-278.

14. Humanes, M. L., Martínez, M., \& Saperas, E. (2013). Periodismo político en España. Prácticas, roles y actitudes. Estudios del Mensaje Periodístico, 19(2), 715-731.

15. Humanes, M. L., Sánchez, M. D., de Dios, R. M., y López-Berini, A. (2013). Pluralism and political parallelism in Spanish television news programmes. Revista Latina de Comunicación Social, 16(68), 566-581.

16. Kepplinger, H. M. (2002). Mediatization of politics: Theory and data. Journal of communication, 52(4), 972-986.

17. Livingston, S., \& Bennett, W. L. (2003). Gatekeeping, indexing, and live-event news: Is technology altering the construction of news? Political Communication, 20(4), 363-380.

18. Martínez-Nicolás, M., \& Humanes, M. L. (2012). Culturas profesionales del periodismo político en España. El discurso de los periodistas sobre la política y las funciones políticas de los medios. En A. Casero (ed.), Periodismo político en España: concepciones, tensiones y elecciones (pp. 47-66). San Cristóbal de la Laguna: Sociedad Latina de Comunicación Social. 
19. Martínez-Nicolás, M., Humanes, M. L., \& Saperas Lapiedra, E. (2014). La mediatización de la política en el periodismo español. Análisis longitudinal de la información política en la prensa de referencia (19802010). Trípodos, 1(34), 41-59.

20. Mazzoleni, G. (2010). La comunicación política. Madrid: Anaya.

21. Mazzoleni, G., \& Schutz, W. (1999). Mediatization of politics: A challenge for democracy? Political Communication, 16(3), 247-261.

22. McNair, B. (2000). Journalism and democracy. London: Routledge.

23. Mellado, C., \& Rafter, K. (2014). Understanding the influence of journalists and politicians on content: A cross-longitudinal analysis of Chilean political news coverage. International Communication Gazette, 76(7), 531-551.

24. Ortega, F. (1994). El mito de la modernización: Las paradojas del cambio social (Vol. 5). Barcelona: Anthropos.

25. Ortega, F. (2003). Una simbiosis compleja. Políticos y periodistas. Telos: Cuadernos de Comunicación, Tecnología y Sociedad, (54), 71-83.

26. Ortega, F. (2011). La política mediatizada. Madrid: Alianza.

27. Ortega, F., Humanes, M. L., Sánchez, C., García Tójar, L., \& Tamarit, A. (2006). Periodismo sin información. Madrid: Tecnos.

28. Patterson, T. E. (1996). Bad news, bad governance. The annals of the American Academy of Political and Social Science, 546, 97-108.

29. Patterson, T. E. (2000). The United States: News in a free-market society. En Gunther, R. \& Mugham, A. (ed.) Democracy and the media: A comparative perspective (pp. 241-265). Cambridge University Press.

30. Salgado, S., \& Strömbäck, J. (2013). Interpretive journalism: A review of concepts, operationalizations and key findings. Journalism, 13(2), 144-161.

31. Strömbäck, J. (2008). Four phases of mediatization: An analysis of the mediatization of politics. The International Journal of Press/Politics, 13(3), 228-246.

32. Strömbäck, J., \& Aalberg, T. (2008). Election news coverage in democratic corporatist countries: A comparative study of Sweden and Norway. Scandinavian Political Studies, 31(1), 91-106.

33. Strömbäck, J., \& Dimitrova, D. V. (2006). Political and Media Systems Matter A Comparison of Election News Coverage in Sweden and the United States. The Harvard International Journal of Press/Politics, 11(4), 131-147.

34. Strömbäck, J., \& Dimitrova, D. V. (2011). Mediatization and media interventionism: A comparative analysis of Sweden and the United States. The International Journal of Press/Politics, 16(1), 30-49.

35. Strömbäck, J., \& Esser, F. (2009). Shaping politics: Mediatization and media interventionism. En Lundby, K. (ed.) Mediatization: Concept, Changes, Consequences (pp, 205-223). New York: Peter Lang Publishing Group.

36. Strömbäck, J., \& Luengo, O. (2008). Polarized Pluralist and Democratic Corporatist Models A Comparison of Election News Coverage in Spain and Sweden. International Communication Gazette, 70(6), 547-562.

37. Strömbäck, J., \& Nord, L. W. (2006). Do politicians lead the tango? A study of the relationship between Swedish journalists and their political sources in the context of election campaigns. European Journal of Communication, 21(2), 147-164. 
38. Strömbäck, J., \& Shehata, A. (2007). Structural biases in British and Swedish election news coverage. Journalism Studies, 8(5), 798-812.

39. Strömbäck, J. et al. (2011). The mediatization and framing of European parliamentary election campaigns. En M. Maier, J. Strömback \& L. Kaid (eds.) Political communication in European parliamentary elections (pp. 161-174). Surrey: Ashgate.

40. van Dalen, A., Albæk, E., \& de Vreese, C. H. (2011). Suspicious minds: Explaining political cynicism among political journalists in Europe. European Journal of Communication, 26(2), 147-162.

41. van Dalen, A., de Vreese, C. H.; \& Albæk, E. (2012). Different roles, different content? A four-country comparison of the role conceptions and reporting style of political journalists. Journalism, 13(7), 903-922.

42. Walgrave, S., \& van Aelst, P. (2006). The contingency of the mass media's political agenda setting power: Toward a preliminary theory. Journal of Communication, 56(1), 88-109.

43. Zaller, J. (1999). A theory of media politics. Manuscript, October, 24. Recuperado de http://www.politics. ubc.ca/fileadmin/user_upload/poli_sci/Faculty/cutler/zaller_theory_of_media_politics.pdf 